\title{
PageRank model of opinion formation on social networks
}

\author{
Vivek Kandiah and Dima L. Shepelyansky \\ Laboratoire de Physique Théorique du CNRS, IRSAMC, Université de Toulouse, UPS, F-31062 Toulouse, France
}

(Dated: April 17, 2012)

\begin{abstract}
We propose the PageRank model of opinion formation and investigate its rich properties on real directed networks of Universities of Cambridge and Oxford, LiveJournal and Twitter. In this model the opinion formation of linked electors is weighted with their PageRank probability. We find that the society elite, corresponding to the top PageRank nodes, can impose its opinion to a significant fraction of the society. However, for a homogeneous distribution of two opinions there exists a bistability range of opinions which depends on a conformist parameter characterizing the opinion formation. We find that LiveJournal and Twitter networks have a stronger tendency to a totalitar opinion formation. We also analyze the Sznajd model generalized for scale-free networks with the weighted PageRank vote of electors.
\end{abstract}

PACS numbers: 05.10.-a,89.20.-a,89.75.-k

\section{INTRODUCTION}

To understand the nature and origins of mass opinion formation is the outstanding challenge of democratic societies [1]. In a last few years an enormous development of such social networks as LiveJournal [2], Facebook [3], Twitter [4] and VKONTAKTE [5], with up to hundreds of millions of users, demonstrated the growing influence of these networks on social and political life. The smallworld scale-free structure of the social networks (see e.g. $[6,7])$, combined with their rapid communication facilities, leads to a very fast information propagation over networks of electors, consumers, citizens making them very active on instantaneous social events. This puts forward a request for new theoretical models which would allow to understand the opinion formation process in the modern society of XXI century.

The important steps in the analysis of opinion formation have been done with the development of various voter models described in a great detail in [8], [9], 10], 11], 12], 13], 14], 15]. This research field became known as sociophysics [8], [10], [12]. In this work we introduce several new aspects which take into account the generic features of the social networks. At first, we analyze the opinion formation on real directed networks taken from the Academic Web Link Database of British universities networks [16], LiveJournal database [17] and Twitter dataset [18]. This allows us to incorporate the correct scale-free network structure instead of unrealistic regular lattice networks, often considered in voter models [13, 14]. At second, we assume that the opinion at a given node is formed by the opinions of its linked neighbors weighted with the PageRank probability of these network nodes. We think that this step represents the reality of social networks: all of network nodes are characterized by the PageRank vector which gives a probability to find a random surfer on a given node as described in [19, 20]. This vector gives a steady-state probability distribution on the network which provides a natural ranking of node importance, or elector or society member importance. In a certain sense the top nodes of PageRank correspond to a political elite of the social network which opinion influences the opinions of other members of the society [1]. Thus the proposed PageRank Opinion Formation (PROF) model takes into account the situation in which an opinion of an influential friend from high ranks of the society counts more than an opinion of a friend from lower society level. We argue that the PageRank probability is the most natural form of ranking of society members. Indeed, the efficiency of PageRank rating is demonstrated for various types of scale-free networks including the World Wide Web (WWW) [19, 20], Physical Review citation network [21, 22], scientific journal rating 23], ranking of tennis players 24], Wikipedia articles [25], the world trade network [26] and others. Due to the above argument we consider that the PROF model captures the reality of social networks and below we present the analysis of its interesting properties.

The paper is composed as follows: the PROF model is described in Sec. II, the numerical results on its properties are presented in Sec.III for British University networks. In Sec. IV we combine the PROF model with the Sznajd model [13, 27] and study the properties of the PROF-Sznajd model. In Sec.V we analyze the models discussed in previous Sections on an example of large social network of the LiveJournal 17. The results for the Twitter dataset [18] are presented in Sec.VI. The discussion of the results is presented in Sec.VII.

\section{PAGERANK OPINION FORMATION (PROF) MODEL DESCRIPTION}

The PROF model is defined in the following way. In agreement with the standard PageRank algorithm [20] we determine the PageRank probability $P_{i}$ for each node $i$ and arrange all $N$ nodes in a monotonic decreasing order of the probability. In this way each node $i$ has a probability $P\left(K_{i}\right)$ and the PageRank index $K_{i}$ with the 
maximal probability is at $K_{i}=1\left(\sum_{i=1}^{N} P\left(K_{i}\right)=1\right)$. We use the usual damping factor value $\alpha=0.85$ to compute the PageRank vector of the Google matrix of the network (see e.g. [19], 20], 28, 29]). In addition to that a network node $i$ is characterized by an Ising spin variable $\sigma_{i}$ which can take values +1 or -1 coded also by red or blue color respectively. The sign of a node $i$ is determined by its direct neighbors $j$ which have the PageRank probabilities $P_{j}$. For that we compute the sum $\Sigma_{i}$ over all directly linked neighbors $j$ of node $i$ :

$$
\begin{array}{r}
\Sigma_{i}=a \sum_{j} P_{j, \text { in }}^{+}+b \sum_{j} P_{j, \text { out }}^{+} \\
-a \sum_{j} P_{j, \text { in }}^{-}-b \sum_{j} P_{j, \text { out }}^{-}, a+b=1,
\end{array}
$$

where $P_{j, \text { in }}$ and $P_{j, \text { out }}$ denote the PageRank probability $P_{j}$ of a node $j$ pointing to node $i$ (incoming link) and a node $j$ to which node $i$ points to (outgoing link) respectively. Here, the two parameters $a$ and $b$ are used to tune the importance of incoming and outgoing links with the imposed relation $a+b=1(0 \leq a, b \leq 1)$. The values $P^{+}$ and $P^{-}$correspond to red and blue nodes respectively. The value of spin $\sigma_{i}$ takes the value 1 or -1 respectively for $\Sigma_{i}>0$ or $\Sigma_{i}<0$. In a certain sense we can say that a large value of parameter $b$ corresponds to a conformist society where an elector $i$ takes an opinion of other electors to which he points to (nodes with many incoming links are in average at the top positions of PageRank). On the opposite side a large value of $a$ corresponds to a tenacious society where an elector $i$ takes mainly an opinion of those electors who point to him.
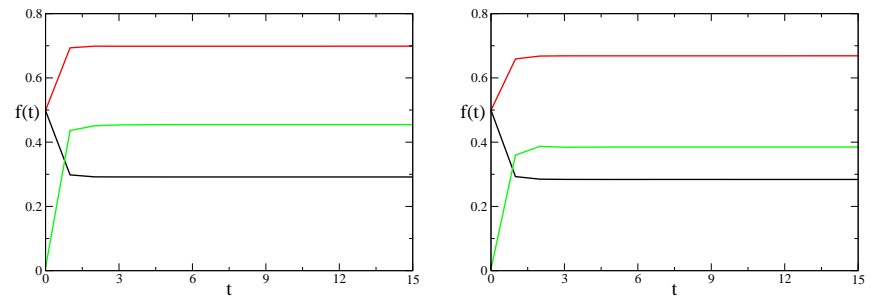

FIG. 1: (Color online) Time evolution of opinion given by a fraction of red nodes $f(t)$ as a function of number of iterations $t$. The red and black curves (top and bottom curves at $t=15$ respectively) show evolution for two different realizations of random distribution of color with the same initial fraction $f_{i}=0.5$ at $t=0$. The green curve (middle curve at $t=15$ ) shows dependence $f(t)$ for the initial state with $N_{\text {top }}$ all red nodes with top PageRank $K$ indexes (highest $P\left(K_{i}\right)$ values, $\left.1 \leq K \leq N_{\text {top }}\right)$. The evolution is done at $a=b=0.5$ and temperature $T=0$. Left panel : Cambridge network with $N_{\text {top }}=2000$. Right panel : Oxford network with $N_{\text {top }}=1000$.

The condition (1) on spin inversion can be written via the effective Ising Hamiltonian $H$ of the whole system of interacting spins:

$$
H=-\sum_{i, j} J_{i j} \sigma_{i} \sigma_{j}=-\sum_{i} B_{i} \sigma_{i}=\sum_{i} \epsilon_{i},
$$

where the spin-spin interaction $J_{i j}$ determines the local magnetic field $B_{i}$ on a given node $i$

$$
B_{i}=\sum_{j}\left(a P_{j, \text { in }}+b P_{j, \text { out }}\right) \sigma_{j},
$$

which gives the local spin energy $\epsilon_{i}=-B_{i} \sigma_{i}$. According to (2), (3) the interaction between a selected spin $i$ and its neighbors $j$ is given by the PageRank probability: $J_{i j}=a P_{j, \text { in }}+b P_{j, \text { out }}$. Thus from a physical view point the whole system can be viewed as a disordered ferromagnet [12, 14]. In this way the condition (11) corresponds to a local energy $\epsilon_{i}$ minimization done at zero temperature. We note that such an analogy with spin systems is well known for opinion formation models on regular lattices [12, [13], 14]. However, it should be noted that generally we have asymmetric couplings $J_{i j} \neq J_{j i}$ that is unusual for physical problems (see discussion in [30]). In view of this analogy it is possible to introduce a finite temperature $T$ and then to make a probabilistic Metropolis type condition [31] for the spin $i$ inversion determined by a thermal probability $\rho_{i}=\exp \left(-\Delta \epsilon_{i} / T\right)$, where $\Delta \epsilon_{i}$ is the energy difference between on-site energies $\epsilon_{i}$ with spin up and down. During the relaxation process each spin is tested on inversion condition that requires $N$ steps and then we do $t$ iterations of such $N$ steps. We discuss the results of the relaxation process at zero and finite temperatures $T$ in next Section.

\section{NUMERICAL RESULTS FOR PROF MODEL ON UNIVERSITY NETWORKS}

Here we present results for PROF model considered on the networks of Cambridge and Oxford Universities in year 2006, taken from [16]. The properties of PageRank distribution $P(K)$ for these networks have been analyzed in [28, 29]. The total number of nodes $N$ and links $N_{\ell}$ are: $N=212710, N_{\ell}=2015265$ (Cambridge); $N=200823$, $N_{\ell}=1831542$ (Oxford) 29]. Both networks are characterized by an algebraic decay of PageRank probability $P(K) \propto 1 / K^{\beta}$ and approximately usual exponent value $\beta \approx 0.9$, additional results on the scale-free properties of these networks are given in [28, 29]. We discuss usually the fraction of red nodes since by definition all other nodes are blue.

The typical examples of time evolution of fraction of red nodes $f(t)$ with the number of time iterations $t$ are shown in Fig. 1. We see the presence of bistability in the opinion formation: two random states with the same initial fraction of red nodes $f_{i}=f(t=0)$ evolve to two different final fractions of red nodes $f_{f}$. The process gives 

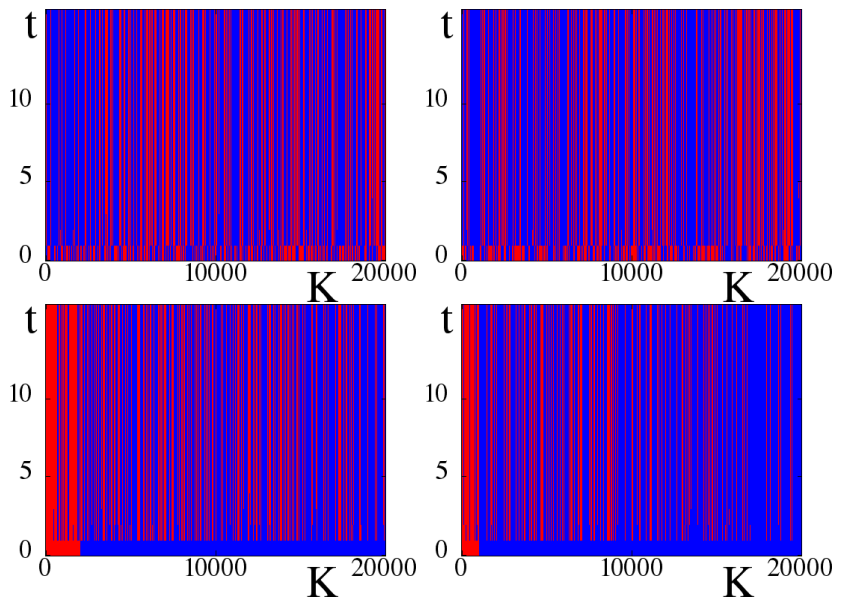

FIG. 2: (Color online) Time evolution of opinion colors (red/gray and blue/black) for the parameters of Fig. 1 left/right column is for Cambridge/Oxford network. The initial fraction of red colors is $f_{i}=0.5$ (top panel) and $N_{\text {top }}$ nodes have red color for bottom panels with $N_{t o p}=2000$ and 1000 for Cambridge and Oxford network respectively. Nodes are ordered by the PageRank index $K$ and color plot shows only $K \leq 20000$.

an impression of convergence to a fixed state approximately after $t_{c} \approx 10$ iterations. A special check shows that all node colors become fixed after this time $t_{c}$. The convergence time to a fixed state is similar to those found for opinion formation on regular lattices where $t_{c}=O(1)$ [13, 14, 32]. The corresponding time evolution of colors is shown in Fig. 2 for first $10 \%$ of nodes ordered by the PageRank index $K$.

The results of Fig. 1 show that for a random initial distribution of colors we may have different final states with \pm 0.2 variation compared to the initial $f_{i}=0.5$. However, if we consider that $N_{\text {top }}$ nodes with the top $K$ index values (from 1 to $N_{\text {top }}$ ) have the same opinion (e.g. red nodes) then we find that even a small fraction of the total number of nodes $N$ (e.g. $N_{\text {top }}$ of about $0.5 \%$ or $1 \%$ of $N$ ) can impose its opinion for a significant fraction of nodes of about $f_{f} \approx 0.4$. This shows that in the frame of PROF model the society elite, corresponding to top $K$ nodes, can significantly influence the opinion of the whole society under the condition that the elite members have the fixed opinion between themselves.

We also considered the case when the red nodes are placed on $N_{t o p}=2000$ top nodes of CheiRank index $K^{*}$. This ranking is characterized by the CheiRank probability $P^{*}\left(K^{*}\right)$ for a random surfer moving in the inverted direction of links as described in [25, 29]. In average $P^{*}\left(K^{*}\right)$ is proportional to the number of outgoing links. However, in this case the top nodes with a small $f_{i}$ values are not able to impose their opinion and the final fraction becomes blue. We attribute this to the fact that the opinion condition (1) is determined by the PageR- ank probability $P(K)$ and that the correlations between CheiRank and PageRank are not very strong (see discussion in [25, 29]).
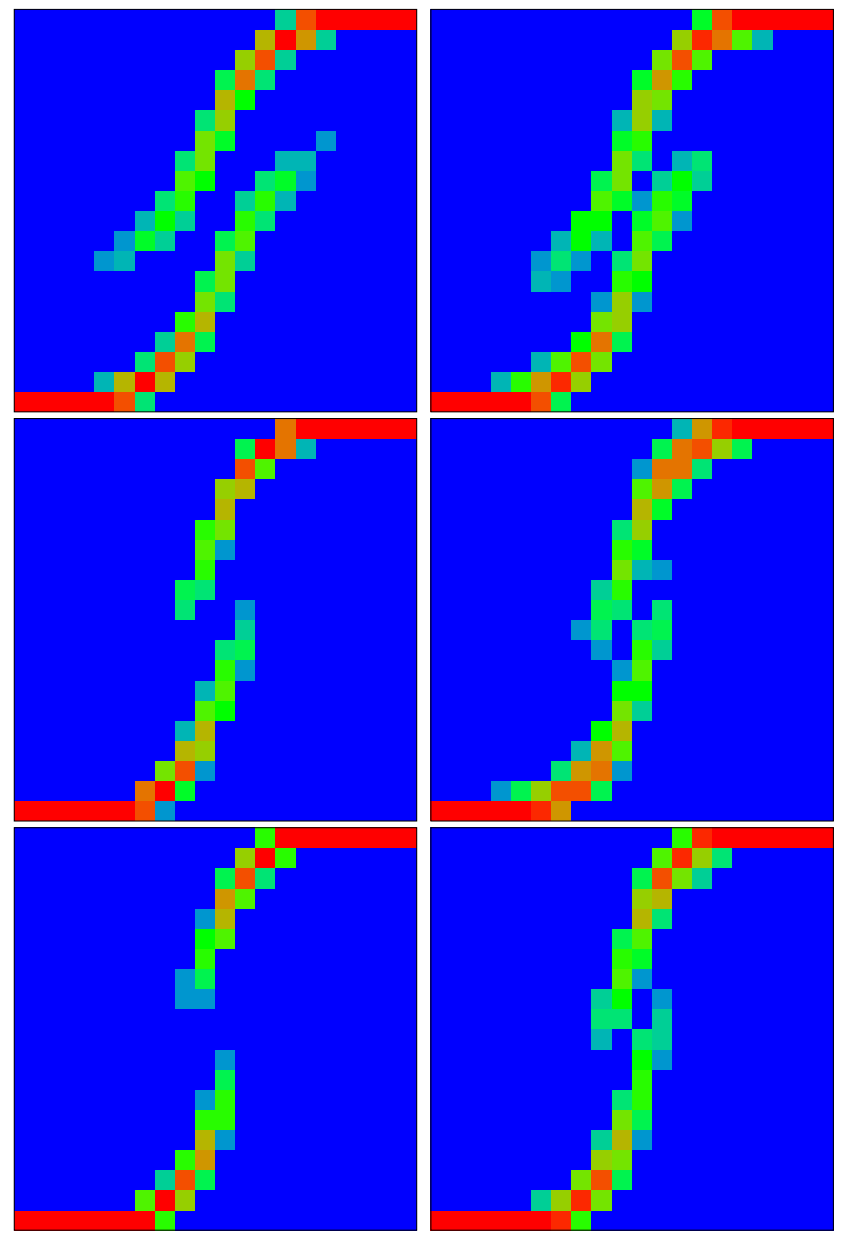

FIG. 3: (Color online) Density plot of probability $W_{f}$ to find a final red fraction $f_{f}$, shown in $y$-axis, in dependence on an initial red fraction $f_{i}$, shown in $x$ - axis; data are shown inside the unit square $0 \leq f_{i}, f_{f} \leq 1$. The values of $W_{f}$ are defined as a relative number of realizations found inside each of $20 \times 20$ cells which cover the whole unit square. Here $N_{r}=$ $10^{4}$ realizations of randomly distributed colors are used to obtained $W_{f}$ values; for each realization the time evolution is followed up the convergence time with up to $t=20$ iterations; here $T=0$. Left column: Cambridge network; right column: Oxford network; here $a=0.1,0.5,0.9$ from top to bottom. The probability $W_{f}$ is proportional to color changing from zero (blue/black) to unity (red/gray).

To analyze how the final fraction of red nodes $f_{f}$ depends on its initial fraction $f_{i}$ we study the time evolution $f(t)$ for a large number $N_{r}$ of initial random realizations of colors following it up to the convergent time for each realization. We find that the final red nodes are homogeneously distributed in $K$. Thus there is no specific preference for top society levels for an initial random distribution. The probability distribution $W_{f}$ of final fractions $f_{f}$ is shown in Fig. 3 as a function of initial fraction $f_{i}$ at 
three values of parameter $a$. These results show two main features of the model: a small fraction of red opinion is completely suppressed if $f_{i}<f_{c}$ and its larger fraction dominates completely for $f_{i}>1-f_{c}$; there is a bistability phase for the initial opinion range $f_{b} \leq f_{i} \leq 1-f_{b}$. Of course, there is a symmetry in respect to exchange of red and blue colors. For small value $a=0.1$ we have $f_{b} \approx f_{c}$ with $f_{c} \approx 0.25$ while for large value $a=0.9$ we have $f_{c} \approx 35, f_{b} \approx 0.45$.

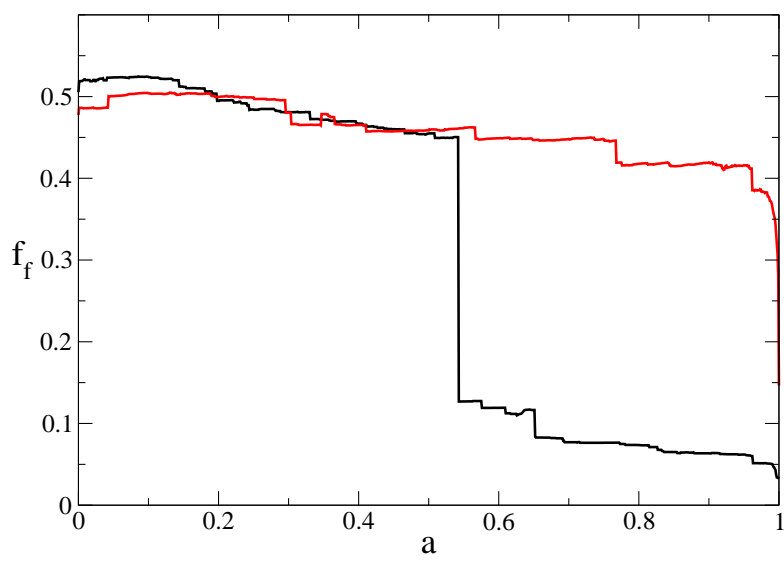

FIG. 4: (Color online) Dependence of the final fraction of red nodes $f_{f}$ on the tenacious parameter $a$ (or conformist parameter $b=1-a$ ) for initial red nodes in $N_{t o p}=2000$ values of PageRank index $\left(1 \leq K \leq N_{\text {top }}\right)$; black and red(gray) curves show data for Cambridge and Oxford networks; here $T=0$.

Our interpretation of these results is the following. For small values of $a \rightarrow 0$ the opinion of a given society member is determined mainly by the PageRank of neighbors to whom he points to (outgoing links). The PageRank probability $P$ of nodes, on which many nodes point to, is usually high since $P$ is proportional to the number of ingoing links [20]. Thus, at $a \rightarrow 0$ a society is composed of members who form their opinion listening an elite opinion. In such a society its elite with one color opinion can impose this opinion to a large fraction of the society. This is illustrated on Fig. 4 which shows a dependence of final fraction of red $f_{f}$ nodes on parameter $a$ for a small initial fraction of red nodes in the top values of PageRank index $\left(N_{t o p}=2000\right)$. We see that $a=0$ corresponds to a conformist society which follows in its great majority the opinion of its elite. For $a=1$ this fraction $f_{f}$ drops significantly showing that this corresponds to a regime of tenacious society. It is somewhat surprising that the tenacious society $(a \rightarrow 1)$ has well defined and relatively large fixed opinion phase with a relatively small region of bistability phase. This is in a contrast to the conformist society at $a \rightarrow 0$ when the opinion is strongly influenced by the society elite. We attribute this to the fact that in Fig. 3] we start with a randomly distributed opinion, due to that the opinion of elite has two fractions of two
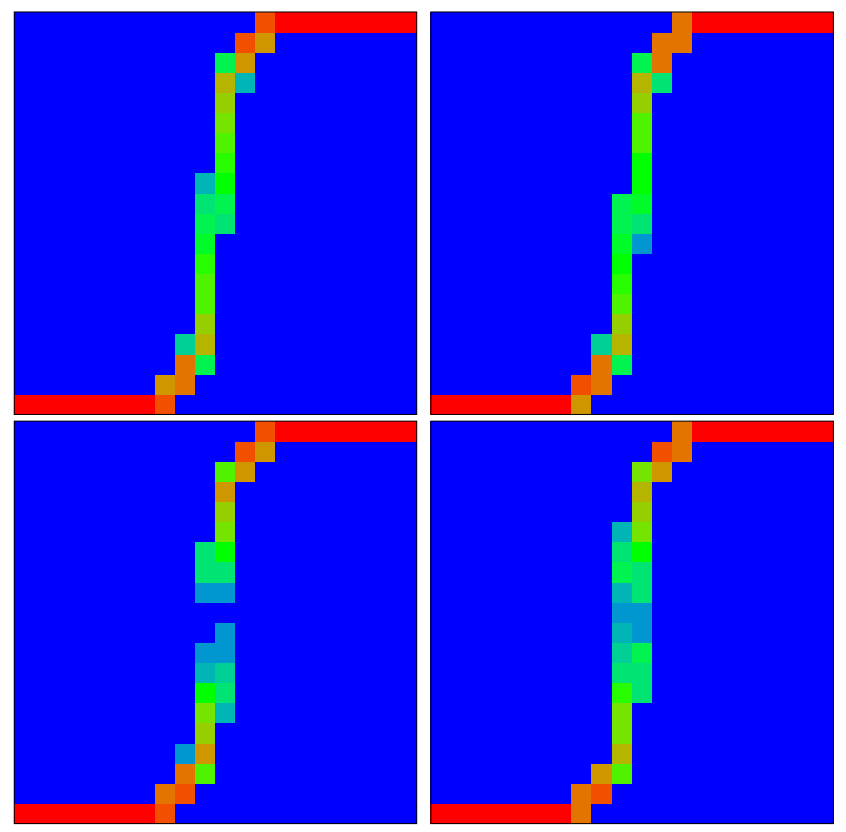

FIG. 5: (Color online) Same as in Fig. 3 (middle panels) at $a=0.5$ but with uniform condition for spin flip being independent of PageRank probability (top panels: $P=1$ in Eq. (10) and PageRank probability $P$ replaced by $\sqrt{P}$ in Eq.(11) (bottom panels); left and right panels correspond to Cambridge and Oxford networks; here $T=0$ and $N_{r}=10^{4}$ realizations are used.

colors that create a bistable situation since two fractions of society follows opinion of this divided elite that makes the situation bistable on a larger interval of $f_{i}$ compared to the case of tenacious society at $a \rightarrow 1$.

To stress the important role of PageRank in the dependence of $f_{f}$ on $f_{i}$ presented in Fig. 3 we show in Fig. 5 the same analysis at $a=0.5$ but for the case when in Eq.(1) for the spin flip we take all $P=1$ (equal weight for all nodes). The data of Fig. 5 clearly demonstrate that in this case the bistability of opinion disappears. Thus the PROF model is qualitatively different from the case when only the links without their PageRank weight are counted for the spin flip condition. We also test the sensitivity in respect to PageRank probability replacing $P$ by $\sqrt{P}$ in Eq.(1) as it is shown in Fig. [5 (bottom panels). We see that compared to the case $P=1$ we start to have some signs of bistability but still they remain rather weak compared to the case of Fig. 3

In fact the spin flip condition (1) can be viewed as a relaxation process in a disordered ferromagnet (since all $J_{i j} \geq 0$ in (2), (3) ) at zero temperature. Such type of analysis of voter model relaxation process on regular lattices is analyzed in [13, 14]. From this view point it is natural to consider the effect of finite temperature $T$ on this relaxation. At finite $T$ the flip condition is determined by the thermal Metropolis probability $\exp \left(-\Delta \epsilon_{i} / T\right)$ as described in previous Section. We follow this thermo- 

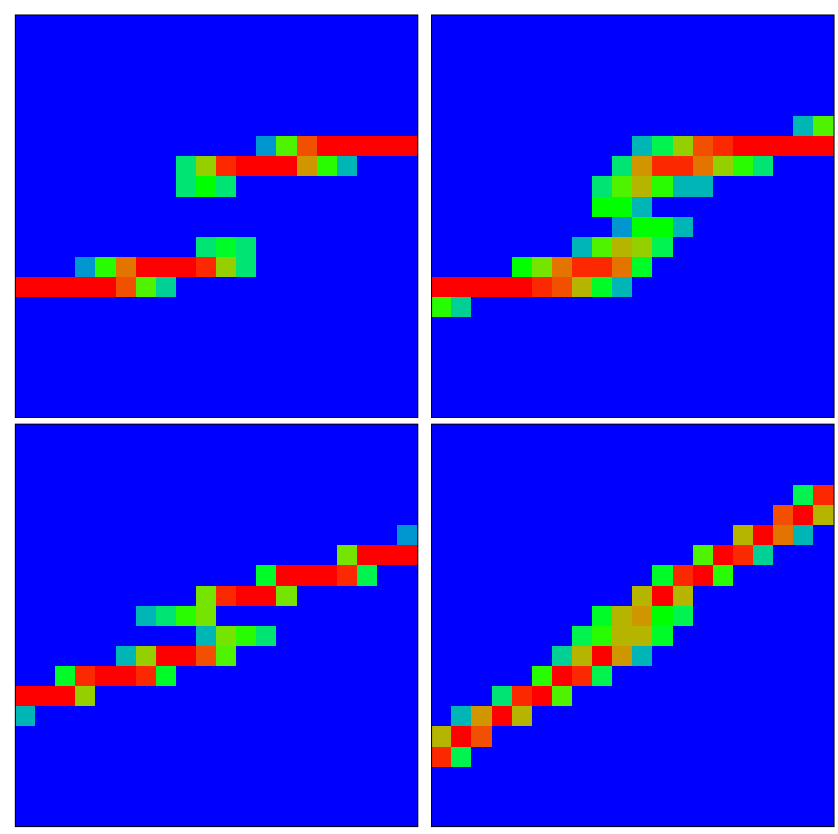

FIG. 6: (Color online) Same as in Fig. 3 (middle panel) at $a=$ 0.5 but at finite temperature $T$ during the relaxation process with $T=0.001$ (top panels) and $T=0.01$ (bottom panels); the number of random initial realizations is $N_{r}=6000$, the relaxation is done during $t=200$ iterations. Left and right columns correspond to Cambridge and Oxford networks.

dynamic relaxation process at finite temperature up to $t=200$ iterations and in this way obtain the probability distribution of final $f_{f}$ fraction of red nodes obtained from initial $f_{i}$ fraction of red nodes randomly distributed over the network at $t=0$. The results obtained at finite temperatures are shown at Fig. 6. They show that a finite temperature $T$ allows to have a finite fraction of red nodes $f_{f}$ when for their small initial fraction $f_{i}$ all final $f_{f}$ were equal to zero. Also the bistability splitting is reduced and it disappears at larger values of $T$. Thus finite $T$ introduce a certain smoothing in $W_{f}$ distribution.
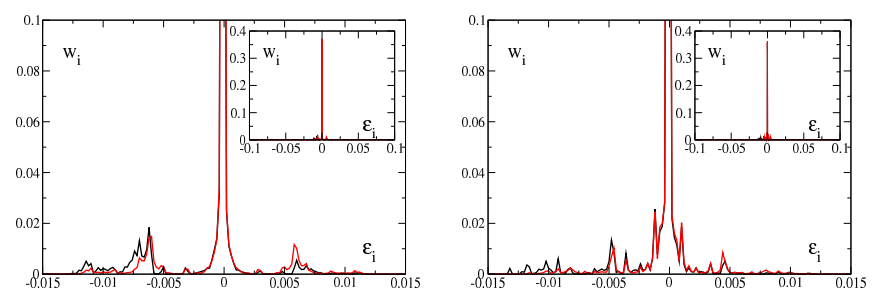

FIG. 7: (color online) Normalized histograms of probability distribution $w_{i}$ over local energies $\epsilon_{i}$ obtained from the relaxation process during $t=10^{3}$ time iterations at temperatures $T=0.01$ (black curve) and $T=0.05$ (red/gray curve); average is done over $N_{r}=200$ random initial realizations. The insets show the distributions on a large scale including all local energies $\epsilon_{i}$. Left and right panels show Cambridge and Oxford networks.
However, the relaxation process at finite temperatures does not lead to the thermal Boltzmann distribution. Indeed, in Fig. [7 we show the probability distribution $w_{i}\left(\epsilon_{i}\right)$ as a function of local energies $\epsilon_{i}$ defined in (2), (3). The distribution $w_{i}\left(\epsilon_{i}\right)$ is obtained from the relaxation process with many initial random spin realizations $N_{r}$. Even if the temperature $T$ is comparable with typical values of local energies $\epsilon_{i}$ we still obtain a rather peaked distribution at $\epsilon_{i} \approx 0$ being very different from the Boltzmann distribution.
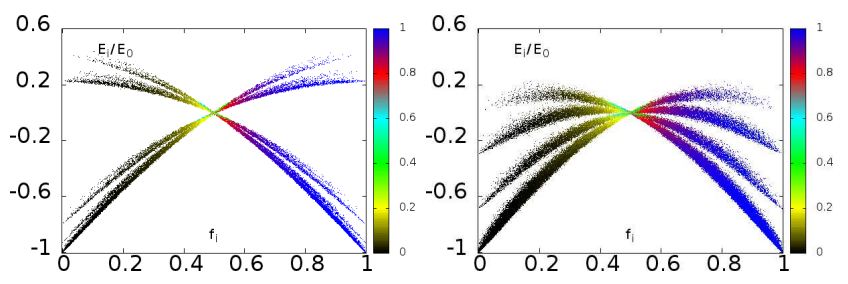

FIG. 8: (Color online) Diagram shows the final fraction of red nodes $f_{f}$, coded by color from $f_{f}=0$ (black) to $f_{f}=1$ (blue/dark gray), as a function of initial fraction of red nodes $f_{i}$ and the total initial energy $E_{i}$; each of $N_{r}>3.5 \times 10^{4}$ random realizations is shown by color point; data are shown after $t=20$ time iterations at $T=0$. The energy $E_{0}$ is the modulus of total energy with all spin up; here $a=0.5$. Left and right panels show data for Cambridge $\left(E_{0}=341.20\right)$ and Oxford $\left(E_{0}=254.28\right)$ networks; bars show color attribution to final probability $f_{f}$.

We argue that a physical reason of significantly nonBoltzmann distribution is related to the local nature of spin flip condition which does not allow to produce a good thermalization on a scale of the whole system. Indeed, there are various energetic branches and probably nonlocal thermalization flips of group of spins are required for a better thermalization. However, the voting is a local process that involves only direct neighbors that seems to be not sufficient for emergence of the global thermal distribution. The presence of a few energy branches is well visible from the data of Fig. 8 obtained at $T=0$. This figure shows the diagram of final fraction $f_{f}$ of red nodes in dependence on their initial fraction $f_{i}$ and the total initial energy $E_{i}=\sum_{m=1}^{N} \epsilon_{m}$ of the whole system corresponding to a chosen initial random configuration of spins. Most probably, these different branches prevent efficient thermalization of the system with only local spin flip procedure. In addition to the above points the asymmetric form of $J_{i j}$ couplings plays an important role generating more complicated picture compared to the usual image of thermal relaxation (see e.g. [30]). We also note that the thermalization is absent in voter models on regular lattices [13. 


\section{PROF-SZNAJD MODEL}

The Sznajd model [27] nicely incorporates the wellknown trade union principle "United we stand, divided we fall" into the field of voter modeling and opinion formation on regular networks. The review of various aspects of this model is given in [13]. Here we generalize the Sznajd model to include in it the features of PROF model and consider it on social networks with their scalefree structure. This gives us the PROF-Sznajd model which is constructed in the following way. For a given network we determine the PageRank probability $P\left(K_{i}\right)$ and PageRank index $K_{i}$ for all $i$ nodes. After that we introduce the definition of group of nodes. It is defined by the following rule applied at each time step $\tau$ :

- i) pick by random a node $i$ in the network and consider the polarization of the $N_{g}-1$ highest PageRank nodes pointing to it;

- $i i)$ if the node $i$ and all other $N_{g}-1$ nodes have the same color (same spin polarization), then these $N_{g}$ nodes form a group whose effective PageRank value is the sum of all the member values $P_{g}=$ $\sum_{j=1}^{N_{g}} P_{j}$; if it is not the case then we leave the nodes unchanged and perform the next time step;

- iii) consider all the nodes pointing to any member of the group (this corresponds to the model option 1) or consider all the nodes pointing to any member of the group and all the nodes pointed by any member of the group (this corresponds to the model option 2); then check all these nodes $n$ directly linked to the group: if an individual node PageRank value $P_{n}$ is less than $P_{\text {group }}$ then this node joins the group by taking the same color (polarization) as the group nodes; if it is not the case then a node is left unchanged; the PageRank values of added nodes are then added to the group PageRank $P_{\text {group }}$ and the group size is increased.

The above time step is repeated many times during time $\tau$, counting the number of steps, by choosing a random node $i$ on each next step. This procedure effectively corresponds to the zero temperature case in the PROF model.

A typical example of the time evolution of the fraction of red nodes $f(\tau)$ in the PROF-Sznajd model is shown in Fig. 9. It shows that the system converges to a steadystate after a time scale $\tau_{c} \approx 10 N$ that is comparable with the convergence times for the PROF models studied in previous Sections. We see that there are still some fluctuations in the steady-state regime which are visibly smaller for the option 2 case. We attribute this to a larger number of direct links in this case. The number of group nodes $N_{g}$ gives some variation of $f_{f}$ but these variations remain on a relatively small scale of a few percents. Here, we should point on the important difference
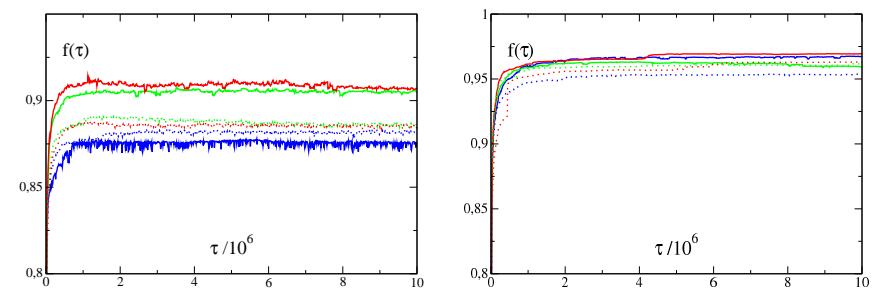

FIG. 9: (Color online) Time evolution of the fraction of red nodes $f(\tau)$ in the PROF-Sznajd model with the initial fraction of red nodes $f_{i}=0.7$ at one random realization. The curves show data for three values of group size $N_{g}=3$ (blue/black); 8 (green/light gray); 13 (red/gray). Full/dashed curves are for Cambridge/Oxford networks; left panel is for option 1 ; right panel is for option 2 .

between PROF and PROF-Sznajd models: for a given initial color realization, in the first case we have convergence to a fixed state after some convergence time while in the second case we have convergence to a steady-state which continue to fluctuate in time, keeping the colors distribution only on average.

The dependence of the final fraction of red nodes $f_{f}$ on its initial value $f_{i}$ is shown by the density plot of probability $W_{f}$ in Fig. 10 (option 1 of PROF-Sznajd model). The probability $W_{f}$ is obtained from many initial random realizations in a similar way to the case of Fig. 3 , We see that there is a significant difference compared to the PROF model (Fig. [3): now even at small values of $f_{i}$ we find small but finite values of $f_{f}$ while in the PROF model the red color disappears at $f_{i}<f_{c}$. This feature is related to the essence of the Sznajd model: here even small groups can resist against totalitar opinion. Other features of Fig. 10 are similar to those found for the PROF model: we again observe bistability of opinion formation. The number of nodes $N_{g}$, which form the group, does not affect significantly the distribution $W_{f}$, we have smaller fluctuations at larger $N_{g}$ values but the model works in a stable way already at $N_{g}=3$. The results for the option 2 of PROF-Sznajd model are shown in Fig. 11 In this case the opinions with a small initial fraction of red nodes $f_{i}$ are suppressed in a significantly stronger way compared to the option 1 . We attribute this to the fact that large groups can suppress in a stronger way small groups since the outgoing direct links are taken into account in this option.

The significant difference between the two options of PROF-Sznajd model is well seen from the data of Fig. 12, Here, all $N_{\text {top }}$ nodes are taken in red (compare with the PROF model in Fig. (4). For the option 1 the society elite succeeds to impose its opinion to a significant fraction of nodes which is increased by a factor 5-10. Visibly, this increase is less significant than in the PROF model. However, for the option 2 of PROF-Sznajd model there is practically no increase of the fraction of red nodes. Thus, 


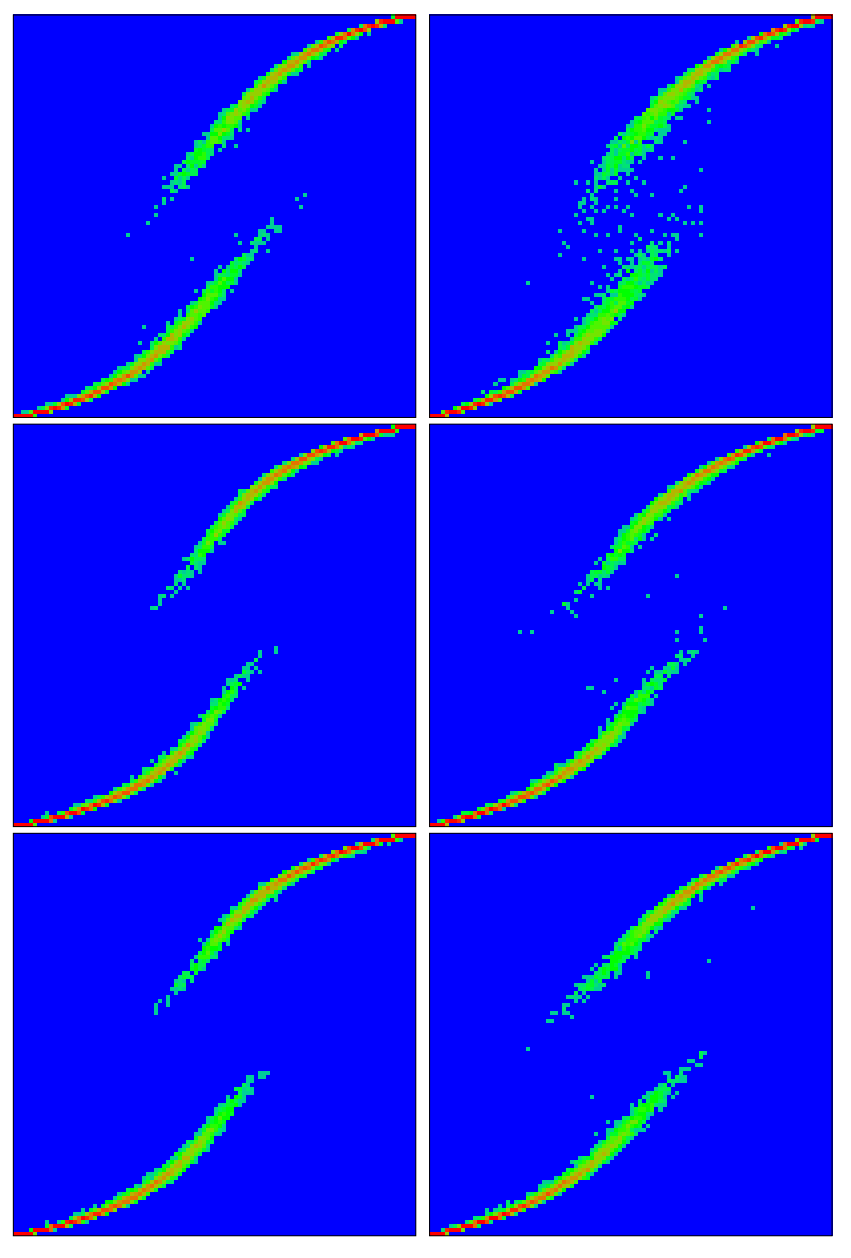

FIG. 10: (Color online) PROF-Sznajd model, option 1: density plot of probability $W_{f}$ to find a final red fraction $f_{f}$, shown in $y$-axis, in dependence on an initial red fraction $f_{i}$, shown in $x$ - axis; data are shown inside the unit square $0 \leq f_{i}, f_{f} \leq 1$. The values of $W_{f}$ are defined as a relative number of realizations found inside each of $100 \times 100$ cells which cover the whole unit square. Here $N_{r}=10^{4}$ realizations of randomly distributed colors are used to obtained $W_{f}$ values; for each realization the time evolution is followed up the convergence time with up to $\tau=10^{7}$ steps. Left column: Cambridge network; right column: Oxford network; here $N_{g}=3,8,13$ from top to bottom. The probability $W_{f}$ is proportional to color changing from zero (blue/black) to unity (red/gray).

in the option 2 the society members are very independent and the influence of the elite on their opinion is very weak.

\section{PROF MODELS ON THE LIVEJOURNAL NETWORK}

Even if one can expect that the properties of University networks are similar to those of the real social networks it is important to analyze the previous PROF models in

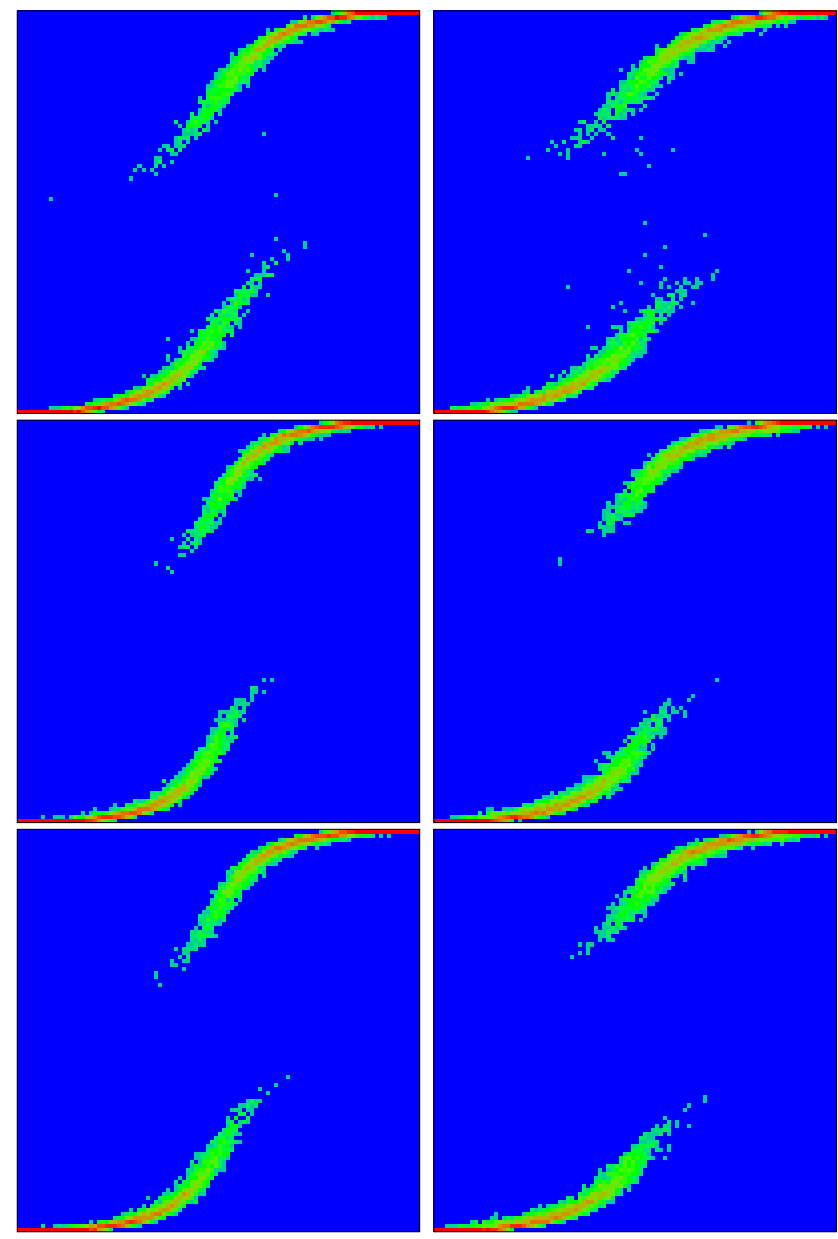

FIG. 11: (Color online) Same as in Fig. 10 but for PROFSznajd model, option 2.

the frame of real social network. For that we use the LiveJournal network collected, described and presented at [17]. From this database we obtain the directed network with $N=3577166$ nodes, $N_{\ell}=44913072$ links which are mainly directed (only about $30 \%$ of links are symmetric). The Google matrix of the network is constructed in a usual way [20] and its PageRank vector is determined by the iteration process at the damping factor $\alpha=0.85$. For the time evolution of fraction of red nodes $f$ we use time iterations in $t$ and $\tau$ defined as in previous Sections.

The PageRank probability decay $P(K)$ is shown in Fig. 13. It is well described by an algebraic law $P(K) \propto$ $1 / K^{\beta}$ with $\beta=0.448 \pm 0.000046$. The convergence of a fraction of red nodes $f(t)$ takes place approximately on the same convergence time scale $t_{c} \sim 5 \sim O(1)$ even if the size of the networks is increased almost by a factor 20.

In a way similar to the University networks we find that the homogeneous opinion of society elite presented in a small fraction of $N_{\text {top }}$ nodes influences a large fraction 

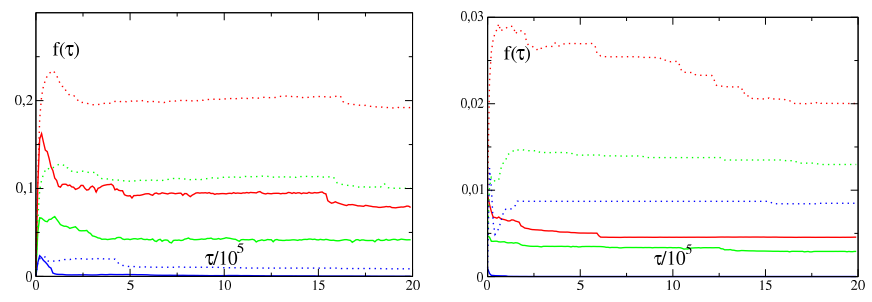

FIG. 12: (Color online) Time evolution of the fraction of red nodes $f(\tau)$ in the PROF-Sznajd model with the initial red nodes for the top PageRank nodes: $N_{t o p}=200$ (blue/black); 1000 (green/light gray); 2000 (red/gray); here $N_{g}=8$. Full/dashed curves are for Cambridge/Oxford networks; left panel is for option 1; right panel is for option 2. Color of curves is red, green, blue from top to bottom at maximal $\tau$ on both panels.
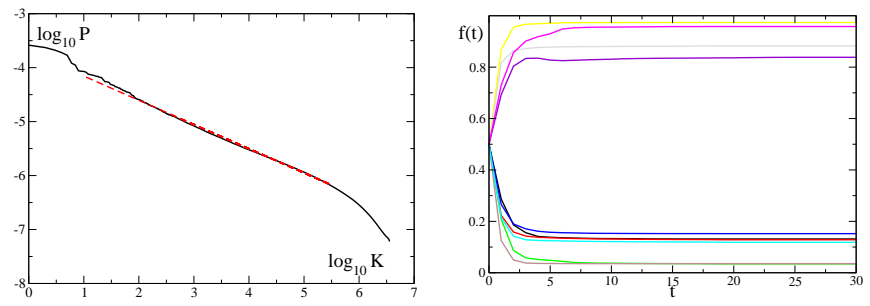

FIG. 13: (Color online) Data for LiveJournal network. Left panel: PageRank probability decay with PageRank index $K$ (full curve), the fitted algebraic dependence is shown by the dashed line $y=b-\beta x$ (for $1 \leq \log _{10} K \leq 5.5$ ) with the exponent $\beta=0.448 \pm 0.000046$ and $b=-3.70 \pm 0.00023$. Right panel: Time evolution of opinion given by a fraction of red nodes $f(t)$ as a function of number of iterations $t$ (cf. Fig. 1) at $a=0.5$, few random initial realizations with $f_{i}=0.5$ are shown.

of the whole society especially when the parameter $a$ is not very large (see Fig. 14 in comparison with Fig. 4). The influence of the elite at $1 \%$ of red nodes is larger in the case of LiveJournal network. It is possible that this is related to a $30 \%$ larger number of links but it is also possible that other structural network parameters also play a role here.

In spite of certain similarities with the previous data for university networks discussed before we find that the opinion diagram for the LiveJournal network (see Fig. 14 right panel) is very different from those obtained for the University networks (see Fig. 31): the bistability practically disappeared. We think that this difference originates from a significantly slower decay exponent for PageRank $P(K)$ in the case of LiveJournal. To check this assumption we compare the probability distribution $W_{f}$ of final opinion $f_{f}$ for an initial opinion fixed at $f_{i}=0.4$ using the PROF model with the usual linear weight $P$ in Eq.(1) and a quadratic weight proportional to $P^{2}$ (see Fig. 15). For the linear weight we find that only very small values of $f_{f} \approx 0.005$ can be found for ini-
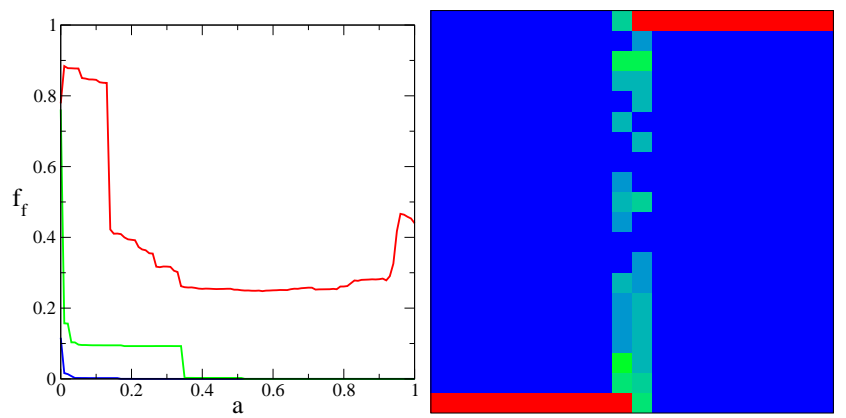

FIG. 14: (Color online) Data for LiveJournal network. Left panel: Dependence of the final fraction of red nodes $f_{f}$ on the tenacious parameter $a$ (or conformist parameter $b=1-a$ ) in the PROF model for initial red nodes in $N_{t o p}$ values of PageRank index $\left(1 \leq K \leq N_{t o p}\right.$, cf. Fig. 4). Here, $N_{t o p}=$ 2000 blue; 10000 green, 35000 red curves (from bottom to top at $a=0.5) ; T=0$. Right panel: Same data as in Fig. 3 at $a=0.5$ with same parameters but for LiveJournal network.

tial $f_{i}=0.4$ while for the quadratic weight we obtain a rather broad distribution of $f_{f}$ values in the main range $0<f_{f}<0.15$ with a few large values $f_{f} \approx 0.6$. Thus we see that the final opinion is rather sensitive to the weight used in Eq.(1). However, in contrast to the University networks (see Fig. 3,Fig. 5), where we have narrow one peak or double peak distributions of $f_{f}$, for the LiveJournal network with quadratic weight we find a rather broad distribution of $f_{f}$. In the spirit of a renormalization map description considered in [10] (see Figs.1,2 there), it is possible to assume that one or two peaks corresponds to one or two fixed points attractor of the map. We make a conjecture that a broad distribution as in Fig. 15 (right panel) can correspond to a regime of strange chaotic attractor appearing in the renormalization map dynamics. In principle, such a chaotic renormalization dynamics is known to appear in coupled spins lattices when threespin couplings are present (see [33] and Refs. therein). It is possible that a presence of weight probability associated with the PageRank in a certain power may lead to a chaotic dynamics which would generate a broad distribution of final opinions $f_{f}$.

We also made tests for the PROF-Sznajd model (option 1) for the LiveJournal database. However, in this case at $f_{i}=0.4, a=0.5$ we found only small $f_{f}$ values (similar of those in Fig. 15, left panel) both for linear and quadratic weights in Eq.(11). It is possible that the Sznajd groups are less sensitive to the probability weight.

\section{PROF MODELS FOR THE TWITTER DATASET}

We also analyzed the opinion formation on the Twitter dataset with $N=41652230, N_{\ell}=1468365182$ taken 

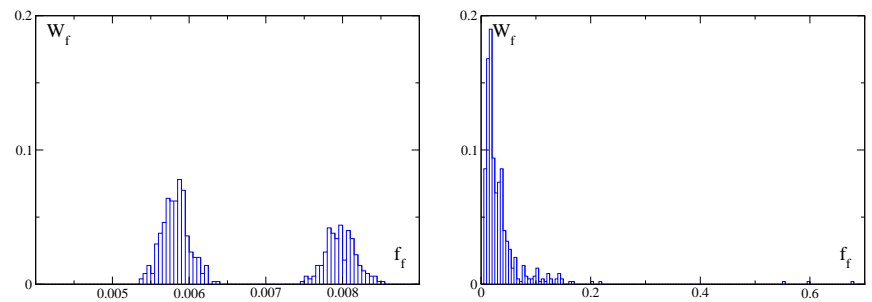

FIG. 15: (Color online) Data for LiveJournal network, probability distribution $W_{f}$ of final opinion $f_{f}$ for a fixed initial opinion $f_{i}=0.4$ and $a=0.5$ in the PROF model. Left panel: usual linear weight $P(K)$ in Eq.(1). Right panel: a quadratic weight $P^{2}(K)$ in Eq.(11). Histograms are obtained with $N_{r}=500$ initial random realizations, the normalization is fixed by the condition that the sum of $W_{f}$ over all histogram bins is equal to unity.

from [18]. This size is rather large and due to that we present only main features of the PROF model for this directed network.
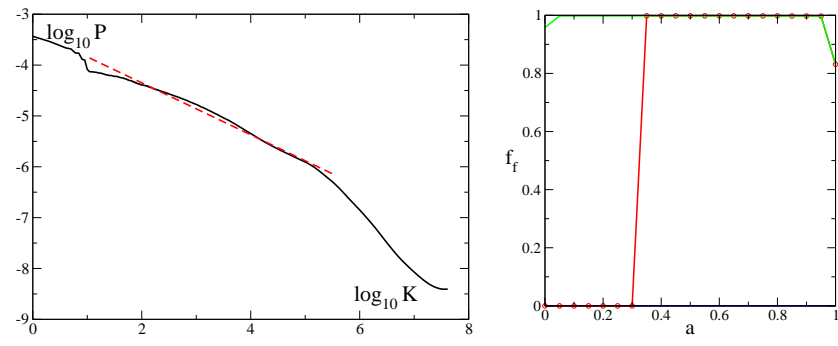

FIG. 16: (Color online) Data for Twitter network. Left panel: PageRank probability decay with PageRank index $K$ (full curve), the fitted algebraic dependence is shown by the dashed line $y=b-\beta x$ (for $1 \leq \log _{10} K \leq 5.5$ ) with the exponent $\beta=0.511 \pm 0.0021$ and $b=-3.33 \pm 0.0069$ (for the range $5.5 \leq \log _{10} K \leq 7$ we find $\beta=1.23$ ). Right panel: Dependence of the final fraction of red nodes $f_{f}$ on the tenacious parameter $a$ (or conformist parameter $b=1-a$ ) in the PROF model for initial red nodes in $N_{t o p}$ values of PageRank index $\left(1 \leq K \leq N_{\text {top }}\right.$, cf. Fig. 4. Fig. 14). Here, $N_{t o p}=1200$ (blue line at $f_{f}=0$ ); 1250 (red curve with circles), 1300 (top gree line); $T=0$.

The dependence of PageRank $P$ on its index $K$ is shown in Fig.16(left panel). For the range $1 \leq \log _{10} K \leq$ 5.5 we find the decay exponent $\beta \approx 0.51$, being similar to those of LiveJournal network (see Fig. 131) even if there is a faster drop of $P$ at larger $K$ values. We note that the value $\beta \approx 0.5$ is rather different from the value usually found for the Zipf law [34] and the WWW [20] with $\beta \approx 1$. It is possible that this is related to a significantly larger average number of links per node which is increased by a factor 3.5 for the Twitter network compared to the University networks analyzed in the previous Sections.

The effect of the homogeneous elite opinion of all red $N_{t o p}$ nodes is shown in Fig.16 (right panel). We see that on the Twitter network a small fraction of elite with the fixed opinion $\left(N_{\text {top }} / N \approx 3 \times 10^{-5}\right)$ can impose it practically to the whole community for all values of the conformist parameter $1-a$. We find that for $N_{t o p}>1300$ all $f_{f}$ values are very close to unity, while for $N_{\text {top }}<1200$ we find $f_{f}=0$ as it is seen in Fig. 16, right panel. Thus, the transition is very sharp. We attribute such a strong influence of elite opinion to the very connected structure of Twitter network with a significantly larger average number of links per node comparing to the University and LiveJournal networks.

At $a=0.5$, for a fixed fraction of initial opinion $f_{i}=0.4$, we find that the probability distribution $W_{f}$ of final opinion $f_{f}$ is located in the range of small values $0.0006<W_{f}<0.0007$ both for linear $P$ and quadratic $P^{2}$ weight used in Eq.(1) (we do not show these data). For the linear weight the situation is rather similar to the case of LiveJournal (see Fig. 15), but for the quadratic weight we find a significant difference between two networks (see Fig. 15). The reason of such a significant difference for the quadratic weight case requires a more detailed comparison of network properties.

The large size of Twitter network makes numerical simulations of the PROF-Sznajd model rather heavy and due to that we did not study this model for this network.

\section{DISCUSSION}

In this work we proposed the PageRank model of opinion formation of social networks and analyzed its properties on example of four different networks. For two University networks we find rather similar properties of opinion formation. It is characterized by the important feature according to which the society elite with a fixed opinion can impose it to a significant fraction of the society members which is much larger than the initial elite fraction. However, when the initial opinions of society members, including the elite, are presented by two options then we find a significant range of opinion fraction within a bistability regime. This range depends on the conformist parameter which characterizes the local aspects of opinion formation of linked society members. The generalization of the Sznajd model for the scale-free social networks gives interesting examples of opinion formation where finite small size groups can keep their own opinion being different from the main opinion of the majority. In this way the proposed PROF-Sznajd model shows that the totalitar opinions can be escaped by small sub-communities. We find that the properties of opinion formation are rather similar for the two University networks of Cambridge and Oxford. However, the results obtained for networks of LiveJournal and Twitter show that the range of bistability practically disappears for these networks. Our data indicate that this is related to a slower algebraic decay of PageRank in these cases com- 
pared to the University networks. However, the deep reasons of such a difference require a more detailed analysis. Indeed, LiveJournal and Twitter networks demonstrate rather different behavior for the $P^{2}$-weighted function of opinion formation. The studies performed for regular networks [10] show existence of stable or bistable fixed points for opinion formation models that have certain similarities with the opinion formation properties found in our studies. At the same time the results obtained in [33] show that three-body spin coupling can generate a chaotic renormalization dynamics. Some our results (Fig. 15, right panel) give indications on a possible existence of such chaotic phase on the social networks.

The enormous development of social networks in a few last years [2 5] definitely shows that the analysis of opinion formation on such networks requires further investigations. This research can find also various other applications. One of them can be a neuronal network of brain which represents itself a directed scale-free network [35]. The applications of network science to brain networks is now under a rapid development (see e.g. [36]) and the Google matrix methods can find useful applications in this field [37].

This work is supported in part by the EC FET Open project "New tools and algorithms for directed network analysis" (NADINE No 288956). We thank A.Benczúr and S.Vigna for providing us a friendly access to the LiveJournal database [17] and the Twitter dataset [18].

[1] J.R. Zaller, The Nature and origins of mass opinion, Cambridge University Press, Cambridge UK (1999).

[2] Wikipedia, LiveJournal, March 9, 2012 http://en.wikipedia.org/wiki/LiveJournal.

[3] Wikipedia, Facebook, March 9, 2012 http://en.wikipedia.org/wiki/Facebook.

[4] Wikipedia, Twitter, March 9, 2012 http://en.wikipedia.org/wiki/Twitter.

[5] Wikipedia, VK (social network), March 9, 2012
http://en.wikipedia.org/wiki/VK\_(social\_network).

[6] S.N. Dorogovtsev and J.F.F. Mendes, Evolution of networks, Oxford Univ. Press (2003).

[7] G. Caldarelli, Scale-free networks, Oxford Univ. Press (2007).

[8] S. Galam, J. Math. Psychology 30, 426 (1986).

[9] T.M. Liggett, Stochastic interacting systems: contact, voter and exclusion processes, Springer, Berlin (1999).

[10] S. Galam, Europhys. Lett. 70, 705 (2005).

[11] D.J. Watts and P.S. Dodds, J. Consumer Research 34(4), 441 (2007).

[12] S. Galam, Int. J. Mod. Phys. C 19, 409 (2008).

[13] C. Castellano, S. Fortunato, and V. Loreto, Rev. Mod. Phys. 81, 591 (2009)

[14] P. L. Krapivsky, S. Redner and E. Ben-Naim, A Kinetic view of statistical physics, Cambridge University Press,
Cambridge UK (2010).

[15] B. Schmittmann and A. Mukhopadhyay, Phys. Rev. E 82, 066104 (2010)

[16] Academic Web Link Database Project http://cybermetrics.wlv.ac.uk/database/

[17] M. Kurucz, A.A. Benczur, A. Pereszlenyi, LargeScale Principal Component Analysis on LiveJournal Friends Network, Proc. Workshop on Social Network Mining and Analysis held in conjunction with 13th ACM SIGKDD International Conference on Knowledge Discovery and Data Mining (KDD 2008), Las Vegas NV, August 24-27 (2008); http://dms.sztaki.hu/en/letoltes/livejournal-data

[18] H. Kwak, C.Lee, H. Park and S. Moon, What is Twitter, a social network or a news media?, Proc. 19th Int. Conf. WWW2010, p.591, ACM, New York, N.Y. (2010); the web data are downloaded from the web site maintained by S.Vigna http://law.dsi.unimi.it/webdata/twitter-2010/

[19] S.Brin and L.Page, Computer Networks and ISDN Systems 30, 107 (1998)

[20] A.M. Langville and C.D. Meyer C D 2006 Google's PageRank and Beyond: The Science of Search Engine Rankings, Princeton University Press, Princeton (2006)

[21] S. Redner, Phys. Today 58(6), 49 (2005).

[22] F. Radicchi, S. Fortunato, B. Markines, and A. Vespignani, Phys. Rev. E 80, 056103 (2009).

[23] J.D. West, T.C. Bergstrom, and C.T. Bergstrom, Coll. Res. Lib. 71, 236 (2010); http://www.eigenfactor.org/

[24] F. Radicchi, PLoS ONE 6, e17249 (2011).

[25] A.O. Zhirov, O.V. Zhirov and D.L. Shepelyansky, Eur. Phys. J. B 77, 523 (2010)

[26] L. Ermann and D.L. Shepelyansky, Acta Phys. Polonica A 120(6A), A158 (2011);

[27] K. Sznajd-Weron and J. Sznajd, Int. J. Mod. Phys. C 11, 1157 (2000).

[28] K.M. Frahm, B. Georgeot and D.L. Shepelyansky, J. Phys, A: Math. Theor. 44, 465101 (2011)

[29] L.Ermann, A.D.Chepelianskii and D.L.Shepelyansky, Towards two-dimensional search engines, arXiv:1106.6215[cs.IR] (2011); http://www .quantware.ups-tlse.fr/QWLIB/dvvadi/

[30] S. Galam and B. Walliser, Physica A 389, 481 (2010).

[31] N. Metropolis, A.W. Rosenbluth, M.N. Rosenbluth, A.H.Teller, and E. Teller, J. Chem. Phys. 21, 1087 (1953).

[32] V. Sood and S. Redner, Phys. Rev. Lett. 94, 178701 (2005).

[33] N.S. Ananikian and S.K. Dallakian, Physica D 107, 75 (1997).

[34] G.K. Zipf, Human Behavior and the Principle of Least Effort, Addison-Wesley, Boston (1949).

[35] V.M. Eguiluz, D.R. Chialvo, G.A. Cecchi, M. Baliki, and A.V. Apkarian, Phys. Rev. Lett. 94, 018102 (2005).

[36] X.-N. Zuo, R. Ehmke, M. Mennes, D. Imperati, F.X. Castellanos, O. Sporns and M.P. Milham, Cereb. Cortex DOI:10.1093/cercor/bhr269 (2011).

[37] D.L. Shepelyansky and O.V. Zhirov, Phys. Lett. A 374, 3206 (2010). 